modèles

linguistiques

\section{Modèles linguistiques}

$76 \mid 2017$

écriture impressionniste et monologue intérieur II

\title{
« Et Dieu se dit en son cœur ». Monologue intérieur hier et aujourd'hui
}

Geneviève Henrot Sostero

\section{OpenEdition}

\section{Journals}

Édition électronique

URL : https://journals.openedition.org/ml/5216

DOI : $10.4000 / \mathrm{ml} .5216$

ISSN : 2274-0511

Éditeur

Association Modèles linguistiques

Édition imprimée

Date de publication : 30 décembre 2017

Pagination : 7-28

Référence électronique

Geneviève Henrot Sostero, « «Et Dieu se dit en son cœur ». Monologue intérieur hier et aujourd'hui », Modèles linguistiques [En ligne], 76 | 2017, document 1, mis en ligne le 02 juin 2019, consulté le 01 juillet 2021. URL : http://journals.openedition.org/ml/5216 ; DOI : https://doi.org/10.4000/ml.5216 


\section{«Et Dieu se dit en son cœur». Monologue intérieur hier et aujourd'hui}

\section{Geneviève Henrot Sostero}

\section{L'évidence de la parole intérieure}

La « parole intérieure » est d'une évidence empirique. Toute notre vie diurne (et nocturne, dans nos rêves) se manifeste par une activité discursive foisonnante, un tourbillon de mots, un puits artésien de répliques sagittaires, un geyser de phrases souvent plus en bribes qu'en morceaux, mais parfois aussi très proches du discours prononcé : brouillons de lettres à jeter plus tard sur le papier, conversations anticipées ou au contraire "révisées » dans l'après-coup de l'esprit d'escalier, reparties émoussées par une langue sept fois tordue et mordue, agenda dressé à l'aube du réveil, débats et enquêtes autour de solutions qui résistent... le tout traversé de paroles écrites, de discours d'autrui et de toutes sortes de bruits trainant alentour, introjectés par une lecture attentive ou une saisie flottante. Comme le narrateur de la Recherche, nous rentrons chez nous "vibrant comme une ruche des propos qui [nous ont] troublés et qui retentissent longtemps en [nous] (Proust 1919 : 185). La parole intérieure n'est d'ailleurs « pas exclusive et la coexistence est de règle dans le dédoublement (ou plus) du circuit verbal mental quand la restitution du discours externe est confrontée au discours poursuivi par l'auditeur qui court deux propos à la fois, écoutant d'une oreille tout en faisant ses commentaires in petto, prolongeant au-dedans les parties et réparties d'un dialogue venu du dehors » (Bergounioux 2001 : 109). Où l'on reconnaît très exactement, par exemple, le propos poétique de Nathalie Sarraute tendu entre conversation et sous-conversation. C'est donc, que nous soyons seul ou en présence d'autrui, un bruissement continu de la langue intérieure (ou des langues, 
entremêlées, alternées, voire amalgamées, pour les polyglottes), une fugue parfois assourdissante, plus ou moins envahissante, qui fait écho ou écran au bourdonnement du " dehors » : discours mental, parole du dedans, endophasie, monologue intérieur, sous-conversation, flux ou courant de conscience, nombreuses sont les dénominations que cette activité a reçues au cours des siècles, n'ayant d'égale que la multiplicité des regards qui lui ont été accordés, révélant tour à tour un intérêt spirituel, moral ou philosophique, un enjeu psychologique, psychanalytique ou littéraire, un objet linguistique, pragmatique ou anthropologique.

Sur cette " sécrétion ininterrompue que l'apprentissage des conduites sociales fixerait dans les formes d'un dialogue intérieur », Gabriel Bergounioux s'interroge en linguiste phonéticien inspiré par Gustave Guillaume (Bergounioux 2001 : 107) : il voit dans l'endophasie (Bergounioux 2004) l'affirmation même de l'existence du locuteur, « l'attestation tangible d'une présence psychique à soi et au monde». Et puisque « rien ne saurait se concevoir d'une 'pensée verbale' qui n'ait, si brève qu'on l'imagine, une signature sonore " (Bergounioux 2001 : 118), cherchant à quantifier ce phénomène dont nul corpus ne permet de débobiner l'enregistrement ${ }^{1}$, il prend pour référent du calcul le locuteur, et pour étalon, le langage sonorisé. Sur le modèle de chiffrages semblables effectués sur la parole prononcée, le calcul de 16 heures vigiles de parole intérieure quotidienne part selon lui de l'ordre de grandeur suivant : « 10 phonèmes/sec. x 3600 secondes/h x 16 heures vigiles/jour égalent 576.000 phonèmes par jour » (Bergounioux, 2001 : 112). Mais ce résultat semble largement sous-estimé, dans la mesure où l'endophasie, exemptée du frein de la gymnastique articulatoire et de la chronématicité acoustique, est de quatre à cinq fois plus rapide que l'exophasie ; que d'autre part, dans notre esprit peuvent se dérouler simultanément plusieurs « fils de paroles "; et qu'enfin ne sont pris en compte ni l'écho verbal intérieur des stimuli langagiers reçus de l'extérieur, ni l'activité

1. Ce rêve qui pourtant court de Stendhal à Sarraute, téléscripteur chez elle, sténographe chez lui : «Supposons qu'un homme pût parler aussi vite qu'il pense et sent, que cet homme une journée entière prononçât de manière à n'être entendu que d'un seul homme tout que qu'il pense et sent, qu'il y eût, cette même journée, toujours à côté de lui un sténographe invisible qui pût écrire aussi vite que le premier penserait et parlerait » (cité par Martin-Achard 2017 : 35). 
langagière du rêve. Bref, la parole intérieure est une noria qui tourne constamment (ou presque) à plein régime et à toute vitesse. Ce que, du reste avait déjà pressenti Victor Egger (1881) dans sa description formelle de la «parole intérieure » (Martin-Achard $2016: 120)^{2}$.

Les années 2000 assistent à un sérieux renouveau d'intérêt pour l'endophasie ${ }^{3}$, après le long monopole que les sciences cognitives des années 1970 s'étaient arrogé à son égard : dans la lignée de Fodor (The Language of Thought, 1975), l'argument de ces dernières était celui d'un langage de la pensée qui fût autonome de toute langue particulière, et grâce auquel l'être humain élaborerait une représentation mentale du monde. Actuellement, un regain d'études linguistiques et littéraires sur l'endophasie s'exprime en France dans quelques publications phares, dont Langue française (2001), Le moyen de parler de Gabriel Bergounioux (2004), «La voix de l'intérieur » de Victor Rosenthal (2012), et, tout récemment, Voix intimes, voix sociales de Frédéric Martin-Achard (2017), auxquelles cette modeste introduction doit beaucoup, et que le présent volume entend prolonger.

\section{Histoire d'une pensée de la parole}

Que la vie intérieure de l'être humain se façonne, en tout ou en partie, sous forme de langage, l'histoire n'a pas attendu notre millénaire pour s'en apercevoir et en philosopher. Dès le XII-VII ${ }^{e}$ avant Jésus-Christ, de nombreux lieux de la Bible font état de propos privés que Jahvé « se dit à lui-même ». Considérant le phénomène avec la «miraculeuse » lucidité analytique qui fut la leur, les Grecs ont même forgé ce terme qui nous est resté : epilegein, « discourir intérieurement à propos de ». Certains passages d'Homère, de Virgile, d'Ovide, de Longus peuvent apparaître comme des monologues intérieurs (Scholes 1966 ; Létoublon 2000). On doit à Claude Panaccio d'avoir retracé l'histoire du « discours intérieur » de l'Antiquité grecque au Moyen-Âge, pour preuve d'une réflexion philosophique concernant cette activité dialogique constante que l'être humain entretient en son for intérieur. Que la pensée soit « un discours

2. La primauté de l'œuvre d'Egger dans la théorisation française moderne du monologue intérieur a fait récemment l'objet de plusieurs études, outre Bergounioux 2004 et Martin-Achard 2017, parmi lesquelles Santone 1998, 1999 et 2009.

3. Voir la périodisation proposée par Martin-Archard $2017: 41$. 
logiquement articulé que l'esprit se tient à lui-même » est la thèse qui traverse toute la tradition philosophique occidentale : elle questionne le rapport entre la pensée et le langage, en quête d'une possible homologie dont on n'a pas encore arrêté l'étymon. Panaccio invite les penseurs du langage et de la pensée, échelonnés sur dix-huit siècles depuis Platon jusqu'à Guillaume d'Ockham, à dévoiler leur position quant à une articulation logique de la pensée et à sa dépendance, ou son indépendance, des langues naturelles. C'est Le Langage de la pensée de Fodor qui a d'abord inspiré à Panaccio (1999), philosophe médiéviste, un parallèle avec la pensée d'Ockham. Or celui-ci apparait comme le point de maturation de toute une histoire de la pensée occidentale du langage intérieur. Aussi Panaccio s'engage-t-il à une telle anabase, afin de « savoir où, comment et pourquoi s'est développée, de Platon à Guillaume d'Ockham, l'idée d'une pensée abstraite et discursive, indépendante des langues mais constituée de signes et dotée comme elles d'une syntaxe et d'une sémantique compositionnelles finement articulées » (Panaccio, 1999 : 26). Les premières traces d'une réflexion théorique sur le discours intérieur se trouvent, disait-on, chez les Grecs : Platon argumente le caractère dialogique de la pensée, cependant qu' Aristote en souligne la logique. S'installe une première dichotomie entre logos endiathtos (discours intérieur) et logos prophorikos (discours extérieur). Les premiers siècles de notre ère développent une conception doctrinale du concept qui culmine chez saint Augustin, et dont la racine théologique jette les pleins feux sur la prière intime, la méditation, l'examen de conscience et la lecture silencieuse des textes sacrés : de cette oratio mentalis ou verbum in corde, les néoplatoniciens voudraient établir si elle relève d'une langue particulière (le latin, en l'occurrence) ou constitue un langage universel. D'Ammonius, de Porphyre et de Boèce, en passant par les Arabes, et se poursuivant chez Thomas d'Aquin, les dominicains et les franciscains, le débat s'enracine et s'envenime, de savoir si la pensée est mère, sœur jumelle ou fille du langage.

La modernité en héritera, par la pratique de l'introspection, un goût pour la méditation philosophique (Montaigne, Pascal) et pour une exposition de l'intériorité subjective (Rousseau). Mais c'est au XIXe siècle que se diversifient les regards convergeant sur la question, à la faveur heuristique : 
- de ses accrochages patents : l'aphasie, la folie, l'onyrologie, soustrayant à la production de pensée le support articulé du langage proféré, semblaient récolter cet irréductible, ce résidu, le propre du langage intérieur ;

- de ses décrochages entre le mot et la pensée : il existerait bien des mots sans pensée (psittacisme) et des pensées sans mots (imagerie) ;

- de son embrayage ontologique : l'apprentissage de la parole (et, ajoutons-nous, l'apprentissage d'une langue étrangère) balaie de feux tournants une relation en formation, avec ses ratés, ses résistances, ses approximations et ses zones d'ombre.

La deuxième moitié du XIXe siècle aura ainsi vu s'affirmer, d'une part, une psychologie plus curieuse de ce qui vient à faire défaut à la relation pensée/langage que de ce qui l'échafaude dans la construction du sujet, et d'autre part, une linguistique incapable « de dépasser la construction d'un système (la langue) pour raisonner l'exercice de son fonctionnement (la parole) »(Bergounioux, 2001 : 18). Car Saussure ne s'adonna à cette passion pour la philosophie du langage que dans les coulisses de ses cours, et nous n'en sûmes rien jusqu'à hier. La philosophie rencontre la psychologie, unissant une remontée à l'intuition platonicienne et à la pratique de l'introspection, pour affirmer, avec le philosophe Victor Egger, que :

À tout instant, lâme parle intérieurement sa pensée. Ce fait, méconnu par la plupart des psychologues, est un des éléments les plus importants de notre existence : il accompagne la presque totalité de nos actes ; la série des mots intérieurs forme une succession presque continue, parallèle à la succession des autres faits psychiques ; à elle seule, elle tient donc une partie considérable de la conscience de chacun de nous (Egger 1881).

Mais La Parole intérieure de Victor Egger resta malencontreusement étouffée sous le boisseau par l'aura et la doxa issues de l'école de médecine en pleine ascension (Broca, Ribot, Charcot, Ballet, Saint-Paul, Netter $)^{4}$, à laquelle on devrait le « lancement » du terme « endophasie ». Comme le résume Martin-Achard (2016: 118), « Le langage intérieur est le champ d'une lutte scientifique féroce entre une tradition matérialiste portée en premier chef par les médecins - aliénistes et neurologues - et un courant spiritualiste,

4. Pour une revue plus détaillée, se reporter à Carroy et alii, $2006: 9-28$, et à Puech $2001: 26-47$. 
héritage psychologique d'une tradition philosophique de l'introspection ». Face à l'observation commune « que la vie psychique est constituée d'une verbalisation continue et silencieuse ", il semble bien « qu'il se joue, entre 1880 et 1900, autour de la question du langage intérieur, ou endophasie, un basculement épistémologique du spirituel au mental, de l'esprit au cerveau » (ibidem).

Si donc Bergounioux qualifie de «négative » cette histoire de la parole intérieure dans les sciences humaines françaises, c'est bien parce que chacune de celles qui s'en sont approchées ne l'a effleurée que pour la rejeter au profit d'un aspect qui s'imposait en rival. C'est ainsi que même Piaget, auteur du Langage et la pensée chez l'enfant (1923), passe à côté « du langage intérieur », qu'il laisse indéfiniment en attente sur le paillasson d'un «sans parler de » : s'il semble être annoncé d'entrée de jeu, ce « chapitre » manquera à son œuvre. C'est finalement de l'étranger, et en particulier de la linguistique allemande (Bühler, 1934) et de l'école russe (Bakhtine, Jakubinski ${ }^{5}, 1923$; Vygotski, 1934 ; le Jakobson de 1929) que viendront les propositions les plus convaincantes.

Si la doxa occidentale des années 1920 posait la fonction psycho-génétique du langage extérieur, dans son rôle de " médium sémiotique » des fonctions psychologiques supérieures et du langage extérieur, en revanche, la pensée linguistique germanophone (Bühler, 1934) et russe (La Parole dialogale Jakubinski, 1923) l'approchent d'un angle de vue sémiotico-descriptif pour en souligner, au contraire, la grande diversité fonctionnelle. Imprégné de Jakubinski, Lev S. Vygotski (1896-1934) consacre le dernier chapitre de Pensée et Langage (1934) à déconstruire trois acceptions en vigueur (Friedrich, 2001) qui voient le langage intérieur comme, respectivement, 1) un réservoir mémoriel des mots, 2) un langage moins les sons, 3) un laboratoire d'élaboration du langage extérieur. Pour sa part, Vygotski revendique la spécificité du langage intérieur comme activité spécifique verbale et non simplement mentale, qui ne serait pas strictement ancillaire du langage extérieur, mais plutôt son symétrique :

Le langage extériorisé est un processus de transformation de la pensée en paroles, sa matérialisation, son objectivation. Le langage intérieur est un

5. Sur un retour à Jakubinski, voir Archaimbault 2012 et Depreto 2013. 
processus de sens inverse, qui va de l'extérieur à l'intérieur, un processus de volatilisation du langage dans la pensée (Vygotski, 1997 : 442-443)

Les interprétations occidentales posthumes de Vygotski ont eu tendance à radicaliser le rôle du langage comme médium sémiotique et moteur psychogénétique des processus psychiques, dans les processus symétriques d'extériorisation et d'intériorisation. Mais retremper la pensée de Vygotski dans son terreau d'origine, les années 20 de l'Union soviétique, apporte d'autres clés de lecture, qui éclairent une démarche plutôt sémiotico-descriptive : Jakubinski avait légué à Vygotski la conviction que le langage est « un ensemble de fonctions verbales variées » (Vygotski, 1997 : 468), dont il importe de décrire les caractéristiques spécifiques et distinctives. La réflexion de Vygotski aboutit à relativiser l'importance du langage extérieur, et en particulier l'incidence de son support phonique, et à promouvoir une approche inductive et descriptive des différentes fonctions du langage. Cet empirisme, il en trouve modèles et témoignages chez des écrivains (Dostoïevski, Tolstoï, Stanislavski), à partir desquels il montre deux grands traits du discours intérieur : sa prédicativité et son abréviation. Nul besoin, en effet, d'expliciter le thème d'un énoncé quelconque si, locuteur et interlocuteur ne faisant qu'un, celui-ci est d'avance présent à l'esprit ; par ailleurs, à l'ellipse des expressions référentielles s'ajoute la réduction à l'os des signes et des structures, du point de vue de leur signifiant. Ce faisant, Vygotski indique une voie d'accès au langage intérieur, parallèle à ce langage égocentrique de l'enfant auquel avait prêté attention Piaget : c'est la pratique du monologue intérieur dans la littérature.

\section{La relève des écrivains}

En France, à la fin du XIXe et au début du XX $X^{e}$ siècle, les écrivains s'emparent de la jachère des sciences en matière de langage intérieur, pour l'arpenter chacun à sa manière.

Les débuts du monologue intérieur en littérature française sont bien connus, des Lauriers sont coupés (1887) d'Édouard Dujardin à son traité sur Le monologue intérieur (1931), en passant par ce « lever de rideau » de Valery Larbaud. À en croire Joyce, leur puissance séminale a fait date. Dans la foulée des études psychologiques du XIXe, 
les romanciers se sont attachés eux aussi à représenter en fiction ce monde intérieur entrevu par la science, dont les manifestations extrêmes, les troubles et les abîmes mettaient soudain en doute et en péril le sentiment d'unité de la personne. $\mathrm{Si}$ «la pensée de la pensée » a promené, de l'Antiquité au XXe siècle, la question du rapport entre pensée et langage, « la parole de la parole » nous amène tout droit à la littérature, rapportant dans les godets de ses aubes un flux de fraîche poésie.

L'histoire du monologue intérieur en littérature française ${ }^{6}$ a reçu de R.-M. Albérès (1966/1972) un premier grand chapitre, cependant que Michel Raimond remonte le courant de certaines prémices et analyse ses différentes acceptions au fil du temps : " né avec l'ambition d'épuiser le contenu total d'une conscience, [le monologue intérieur] s'affirme de plus en plus comme le moyen de montrer comment le réel apparaît à une conscience » (Raimond, $1985: 274)^{7}$. Répondent à l'appel plusieurs générations d'écrivains, des années 1930 (Schlumberger, Daniel-Rops, Jouve, Bopp, Bert, Bloch, Cohen) à l'immédiat après-guerre sous l'influence de Joyce, Woolf, Faulkner, Kafka, et au Nouveau Roman (Sarraute, Beckett, Robbe-Grillet, Butor, Pinget, Simon). Mais un nouveau chapitre gagnerait à s'attacher aux transformations contemporaines du monologue intérieur, comme le montre éloquemment F. Martin-Achard dans ses Voix intimes, voix sociales (2017), chez François Bon, Laurent Mauvignier, Jacques Serena, ou encore, mêlé à d'autres techniques narratives, chez Bernard Noël, Régis Jauffret, Tanguy Viel, Matthias Énard, Emmanuel Adely, Hélène Lenoir, Tristan Garcia, Nicole Caligaris, Luc Lang...

\section{Variations sur le thème et sur la forme}

Le monologue intérieur a été tour à tour envisagé dans sa substance (un certain flux de pensées) et dans sa forme (abréviation, prédication,

6. Des commentaires plus précoces apparaissent chez Lämmert, 1955, Bauformen des Erzählens, Stuttgart, 1955, chez Franz Stanzel, 1955, , « Die typischen Erzählsituationen ", Roman dargestellt an Tom Jones, Moby-Dick, The Ambassadors, Ulysses, Vienne, 1955.

7. Si la notion de «monologue intérieur » se rencontre çà (Dumas 1845) et là (Bourget 1892), et si la pratique d'un certain monologue intérieur (mais introduit par un modus-dire) se rencontre chez Stendhal, Sand ou Hugo, c'est le critique russe Chernyshevski (1856) qui, le premier, lui associe la définition qui nous intéresse, à propos de Tolstoï (Struve 1954). 
ellipse, rythme ...). Les narratologues en ont souligné la révolution particulière, à savoir la progressive disparition du narrateur primaire et, partant, la modification profonde des rapports entre instances narratives et instances actoriales (Hamburger 1977), la distinction entre récit de paroles et récit de pensées (Banfield 1982), et la double triade des techniques de rapport (discours rapporté, discours transposé, discours narrativisé : Genette 1972; monologue rapporté, monologue narrativisé, psycho-récit : Cohn 1978), ou des tentatives de systématisation des discours ou styles (direct/indirect, libre/régi : Rosier 1999). Les linguistes et stylisticiens y recensent des faits d'expression touchant à la problématique énonciative des rapports entre discours citant et discours cité, la présence ou l'absence de verbe introducteur (modus dicendi), de ponctuation signalétique (tirets, guillemets), la prégnance de l'interprétation du point de vue (Rosier 1999, Philippe 1997-2009, Rabatel 2001, 2008). Parmi les contradictions intrinsèques aux discours critiques, tiraillés entre deux positionnements (philosophique versus linguistique), celle qui touche au bras de fer subi par le monologue intérieur entre réalisme mimétique et pacte de communication a évolué avec le temps (au cours du siècle) à la faveur d'exercices de lecture qui, passés par la cure d'amaigrissement du Nouveau Roman, ont peu à peu habitué le lecteur à faire son deuil des paramètres explicites et rationnels de la narration : logique temporelle ordonnée, mimétisme descriptif, identification discrète des instances énonciatives, repérage univoque du point de vue.

Dès les années 1950, force est à Struve (1954), Humphrey (1951, 1968), comme plus tard à Steinberg (1978), Cohn (1978), Banfield (1981), Burunat (1980) ou Coelho de Carvalho (1981), d'admettre une évidence : chaque auteur s'invente une forme à soi de monologue intérieur, adaptée à son projet poétique. On peut cependant tenter hardiment d'extraire de ces pratiques quelques constantes qui, impliquées avec plus ou moins d'audace et d'extrémisme, composent dans chaque cas une formule originale, dont la recevabilité et la réussite tiennent à l'équilibre obtenu entre différents pôles. Un faisceau de tensions oscille en effet :

- entre vérité et mensonge : une mimésis de la vie intérieure est-elle possible sans distorsions mensongères?

- entre compréhension et illisibilité : une pure mimésis du monologue 
intérieur, privée de toute explicitation référentielle, pourra-t-elle jamais être compréhensible à un lecteur autre?

- entre contenus langagiers et contenus iconiques de la pensée : comment faire pour transposer en langage la part iconique de la pensée ? N'est-ce pas trahir d'emblée le projet mimétique ? Peut-on considérer que le langage régisse toutes les formes de vie psychique (Bergounioux, 2001) ou bien, selon la définition jamesienne du courant de conscience, avec l'endophasie proprement langagière se trouvent charriés toutes sortes d'autres ingrédients échappant aux structures du langage : sentiments, sensations, perceptions, intuitions?

Cependant, un certain consensus s'est cristallisé autour d'une « technique » s'efforçant de rendre dans sa vérité spontanée un courant continu de conscience, par des traits tour à tour :

- narratologiques : l'absence de toute marque manifestant l'intervention d'un auteur ou narrateur hétérodiégétique (Genette 1972 : 193 ; Cohn $1984: 10$ ) ; l'hégémonie du « je » (Weissman 1978 : 60) ; le dispositif de la communication (Sallenave 1972 ; Francoeur 1978, Joly 1987 ; Philippe 2001a-b, 2017 ; Rabatel 2008) ;

- temporels : temps présent coïncidant à l'énonciation narrative (par opposition au régime autobiographique « en différé ») (Genette 1972 ; Cohn 1984) ;

- stylistiques : abondance de phrases nominales, de verbes à l'infinitif, prégnance de la ponctuation, ruptures syntaxiques et ellipses (Humphrey 1968), prédominance de phrases exclamatives, absence presque totale de formes verbales de la narration et de la description, opacité de la référence (Cohn 1984 : 252-263 ; Philippe 2009).

Pour autant, l'effective et concrète variété des techniques personnelles empêche de tracer un portrait-robot d'un monologue intérieur unique, et oblige à conserver un certain effilochage de ses contours. Ce qui rend très difficile, et toujours contestable, une systématisation de ses formes (Martin-Achard 2017 : 23 et ss.), sauf à s'arrêter à la synthèse suffisamment générique pour embrasser une multiplicité de poétiques apparentées : « une parole intérieure, 
muette et auto-adressée, affranchie de toute instance narrative sur le plan énonciatif et cherchant sur le plan stylistique à figurer le discours de la pensée » (ibidem $2017: 37$ )

Depuis William James (1890), l'idée a fait son chemin, d'une activité psychique intérieure profonde comme un iceberg, dont seule la pointe serait confiée à une structure langagière. Sans doute fautil considérer cette multiplicité d'ingrédients non seulement comme potentiellement coïncidents (j'entends, je vois, j'éprouve et je pense parler tout ensemble), mais aussi comme se chevauchant partiellement sur une échelle continue qui irait, en termes sarrautiens pour faire plus vite, du tropisme à la sous-conversation et de celle-ci à une conversation. La frontière entre l'intérieur et l'extérieur ne tenant pas tant à une phonicité ou non de cette production (on s'entend penser et lire comme on entend intérieurement la voix des autres) qu'à son articulation sonore effective, qu'à son acoustique. Trois cas de figures semblent témoigner de la fragilité de cette frontière entre intérieur et extérieur : 1) le langage égocentrique de l'enfant (étudié par Piaget) ; 2) le dialogue volontiers elliptique, télégraphique, «à demi-mots » de qui partage présupposés, implicites, référents et histoire conversationnelle ${ }^{8}$, et qui possède des traits communs avec le langage intérieur ; et inversement 3) des pensées tellement turbulentes et bruyantes qu'elles tournent en un soliloque que tous décrient, quand tous les pratiquent à l'occasion, sans même s'en rendre compte. La frontière est labile entre dedans et dehors, tant il est facile qu'une excitation plus grande donne voix à cette conversation muette :

Charles avait beau se remuer, aller et venir, il était poursuivi par un monologue intérieur, dont quelques phrases montaient de temps à autre jusqu'à ses lèvres et faisaient retourner les passants ${ }^{9}$.

Les représentations de l'endophasie varient donc, selon l'époque et l'auteur, sur plusieurs axes, en termes tant de contenu que de forme.

8. Car si la pensée et le langage intérieur semblent désorganisés, c'est, non point par comparaison au dialogue extérieur spontané, quotidien, mais plutôt par rapport au discours littéraire et au dialogue tel que la littérature l'a poli jusqu'il y a peu, pour le sertir dans ses autres séquences textuelles. C'est ce que révèlent les études consacrées à la langue parlée.

9. Citation de Goncourt (Ch. Demailly, $1860: 208)$ fournie comme exemple de monologue intérieur par le Trésor de la langue française. 
D'une part, l'étiquette de «monologue intérieur » peut paraitre étroite à d'aucuns, de par sa référence à cette couche supérieure de l'activité psychique la plus proche de l'exophasie, à chaque fois que l'écrivain entend inclure, dans ce tourbillon muet, des sensations, des pulsions, des images dépourvues de paroles (le tropisme de Nathalie Sarraute). On préférerait alors l'expression plus compréhensive de courant de conscience ou de flux de conscience jamesien qui, embrassant toute l'activité psychique consciente, ne se limite pas à sa couche articulée en langue.

D'autre part, la distinctivité formelle entre endophasie et exophasie représentées en littérature évolue selon le repoussoir que la seconde constitue pour la première. À l'époque de Dujardin, pouvait sembler révolutionnaire une phrase légèrement fragmentaire, délicatement elliptique, là où la pratique du dialogue littéraire n'avait pas encore pris la mesure exacte d'un semblable désordre syntaxique dans la vraie parole prononcée. Aujourd'hui que l'étalon de l'endophasie n'est plus le dialogue littéraire (construit et maîtrisé), mais la langue parlée dans toutes ses approximations, ses accrocs et ses ellipses, il en faut beaucoup plus pour qu'endophasie et exophasie se campent distinctement sur les berges opposées du discours. Et l'on est bien moins convaincu qu'il n'existe pas une sorte de continuum entre la parole du dedans et la parole du dehors (dans son jaillissement impulsif, par exemple). Comme le remarque Bergounioux (2011 : 122), «L'endophasie ne semble différer de la parole explicite ni par sa grammaire, ni par son lexique, à la réserve d'un emploi généralisé de l'asyndète et de l'anaphore, et d'une surreprésentation de la prédicativité », sauf à considérer que, « [a]ppliquée à l'endophasie, la conversion de la perception en une séquence linguistique s'avère identique à celle de l'exophasie, si ce n'est que : 1) le locuteur et l'auditeur sont confondus, 2) le signal est virtuel, 3) pour l'auditeur, livré à son propre discours, entendre et comprendre ne sont pas distinguables » (ibidem).

\section{Dujardin, avant, après}

Le présent volume «tourne autour » de Dujardin, dans tous les sens du terme. S'il l'aborde à nouveaux frais, c'est pour mieux faire 
entendre un chœur de voix en canon, chacune ayant « son » monologue intérieur, «sa » propre formule stylistique pour rendre une voix intérieure dans toute son idiosyncrasie. C'est pour mieux montrer, aussi, que ce phénomène que décrivaient déjà les Anciens n’a pas attendu Dujardin, ni le terroir français, pour germer et fructifier en littérature. Ainsi, Marjorie Sabbatorsi montre combien l'Anglais Anthony Trollope (1815-1882), dans son He Knew He Was Right (1869), tenait à représenter le monde quotidien tel qu'il est filtré par une conscience, ses pensées, ses perceptions les plus fugitives. Et il avait déjà jeté la gourme réaliste du roman psychologique, en laissant planer le doute sur les ancrages énonciatifs, de telle sorte que le lecteur, privé impromptu des didascalies du narrateur, est périodiquement plongé dans un concert de voix qui se mêlent et se confondent.

L'article d'Yvonne Heckmann, envisage la teneur musicale du monologue intérieur, en particulier en raison de la mouvance wagnérienne dont il témoigne à ses débuts. Les ceps français du flux de l'intériorité ne sont pas non plus les seuls inventifs en matière de mariage entre littérature et musique. Musicienne, musicologue et mélomane, Yvonne Heckmann, très sensible aux rythmes du langage comme à la présence du thème musical en littérature, analyse chez l'Autrichien Arthur Schnitzler (1862-1931) la fécondation de l'orchidée Dujardin par le bourdon de la musique. Elle décèle dans les deux nouvelles Leutnant Gustl et Fräulein Else l'évolution du leitmotiv wagnérien vers une forme d'écriture réticulaire rhizomatique, proliférante, destinée à dire et mimer l'implosion intérieure du moi.

Non que la venue au jour du monologue intérieur en France soit ici proprement « contournée » au profit d'un élan centrifuge. Mais on cherche à en montrer l'aura, le rayonnement, tantôt rétrospectivement, en filant des racines d'Outre-mer, d'Outre-Manche ou d'Outre-Rhin ; tantôt, féconde descendance, en montrant la mise en exergue de sa pratique au début du $X X^{\mathrm{e}}$ siècle et sa reprise dans la deuxième moitié du $X X^{e}$ siècle. C'est bien « autour » de Dujardin que gravitent ainsi les études d'André Joly, attaché à Valery Larbaud, et de Dairine O'Kelly, qui revient sur James Joyce. 
Quoique le présent numéro soit consacré au monologue intérieur, ce n'est pas sur Molly Bloom que revient ici Dairine O'Kelly, mais sur les paramètres formels et thématiques qui tissent, selon une logique fractale, les différents niveaux d'architecture d'Ulysse : le monologue intérieur, d'ailleurs rarement utilisé "à l'état pur », n'est qu'un des moyens de structuration du texte, parmi lesquels dominent un « dédale de voies » (structuration spatio-temporelle à la recherche d'un amalgame) et une " polyphonie de voix " (structure énonciative complexe où pointent, entre autres, plusieurs sortes de monologues intérieurs). Le tout sur le fond, essentiel, d'une architecture d'ensemble qui permet de lire les diverses cartes superposées des hypotextes (mythique, biblique) et de l'hypertexte joycien, tant dans le sens genettien (copie supérieure et lisible du palimpseste) que dans le sens informatique contemporain (réseau de renvois et de liens intratextuels tissés par des nœuds de mots en écho).

André Joly revient quant à lui sur le rôle de première importance qu'a joué Larbaud entre Dujardin et Joyce. Faisant une large place à l'investigation génétique, sa comparaison des diverses pratiques du monologue intérieur par Larbaud, d'une nouvelle l'autre, de 1920 à 1923, illustre la géométrie variable qui caractérise cette variante spéciale du type de texte dialogal, et nous convainc du fait qu'il faille en retenir, tout au plus, une loi de prototype, voire un principe galactique de variation infinie : «à chacun ses monologues intérieurs ». On pourraît dès lors se demander, par-delà toutes les synthèses qui en ont été faites de par le passé (Humphrey 1968) ou plus récemment (Philippe 2001, Martin-Achard 2017), s'il ne faudrait pas revoir en termes, non stylistiques, mais seulement poétiques, le noyau dur du monologue intérieur : de même que le régime de la parole proférée connait des styles variables à l'infini, selon les caractères, les facondes et les prestances, de même le monologue intérieur n'a guère de raison d'apparaître moins varié. Ce qu'Egger soupçonnait déjà, quand il renvoyait dos à dos la brevitas de l'un et la copia de l'autre.

Pierre-François Peirano met en lumière l'utilisation encore trop méconnue du monologue intérieur dans les romans policiers de 1'Américain Raymond Thornton Chandler (1888-1959). Étayée des 
réflexions théoriques menées par l'auteur, la technique narrative qui donne vie à Philip Marlowe immerge le lecteur dans les pensées du détective-narrateur, dont le regard et les opinions colorent de subjectivité critique la saisie crue de la société américaine des années 1940 et 1950. Mais l'auteur ne lance-t-il pas au lecteur un défi qui devrait le rendre, sinon méfiant, du moins prudent face à cette ouverture excessive d'une pensée privée ? Ainsi la question du monologue intérieur mène-t-elle au débat entre réalité et illusion et trouve même un écho dans les films inspirés par les romans de Chandler.

Laure Levêque voit dans La Semaine sainte (1958) de Louis Aragon un tournant de l'œuvre, en rupture d'avec le cycle du Monde réel, et qui attend du monologue intérieur et du travail sur l'énonciation un déblocage authentifiant de la parole, qui lui permettra d'exprimer, à travers le personnage homologue de Géricault, les vicissitudes d'un artiste tourmenté par l'histoire de son temps.

Enfin Nathalie Vincent-Aroux (Monologue intérieur et éclats impressionnistes : traduction du début de "Bedsitter ») expose les problèmes liés à la traduction du début d'un des récits impressionnistes d'Eva Figes (1932-2012).

\section{Bien après Dujardin : le monologue intérieur aujourd'hui}

Sans doute la seule vocation romanesque de mimesis d'expérience suffirait-elle à expliquer la permanence (ou tout au moins la résurgence périodique), tout au long du XXe et du XXI e siècles, de propositions esthétiques promouvant le monologue intérieur. Le solipsisme croissant de notre ère, l'écartèlement toujours plus frappant entre, d'une part, l'omniprésence de supports de communication et d'autre part, la fragilité et la rareté toujours plus cruciales d'une communication « profonde » laissent entrevoir quelques raisons de cet enfermement des voix dans le carcan d'une intériorité toujours plus étanche : que savoir, que dire d'autre de l'être humain que ce qui l'habite seul au monde, que ce qui ne peut lui manquer, quand bien même viendraient à faire défaut ces relations sociales, privées ou publiques, qui constitue l'humanité en société ? Rien d'étonnant que la littérature du troisième Millénaire vienne multiplier les romans du for intérieur. 
La littérature de l'extrême contemporain semble ainsi avoir fait entièrement sienne la prescription wagnérienne de Théodor de Wyzewa en 1886, pour qui le roman devait désormais montrer le monde filtré par une seule et même conscience intérieure :

Le romancier dressera une seule âme, qu'il animera pleinement : par elle seront perçues les images, raisonnés les arguments, senties les émotions : le lecteur, comme l'auteur, verra tout, les choses et les âmes, à travers cette âme unique et précise, dont il vivra la vie. L'artiste devra limiter la durée de la vie qu'il voudra construire. Il pourra ainsi, durant les quelques heures de cette vie, restituer tout le détail et tout l'enchaînement des idées. On n'aura plus des perceptions isolées, inexpliquées, mais la génération même, continue, des états mentaux ${ }^{10}$.

$\mathrm{Ce}$ « retour en spirale » à la vie intérieure appelle un retour à l'analyse de ses modalités poétiques. Sur ce filon, qu'il cultive depuis quelques années, Martin-Achard observe combien :

tout un pan de la prose narrative actuelle se caractérise par une prépondérance accordée à la vie psychique des personnages et par la multiplicité des modes de représentation de cette intériorité, mêlant, parfois au sein d'une même phrase, les catégories distinguées par Cohn (monologue intérieur, monologue narrativisé, psycho-récit) à des phénomènes de point de vue. (Martin-Achard, $2016: 1$ )

On en trouvera une exploration récente dans Voix intimes, voix sociales. Usages du monologue romanesque aujourd'hui (MartinAchard, 2017), qui invite à déployer l'éventail de lectures de la modernité en clé de monologue intérieur. Étudiant la « refondation du monologue intérieur dans le roman français à partir des années 1980 », l'ouvrage met en lumière « la tension entre l'intime et le social qui parcourt les monologues contemporains » et invite, comme ce numéro de Modèles linguistiques, à explorer les mondes bruissants et silencieux des anti-héros d'aujourd'hui.

Université de Padoue

\section{Bibliographie}

AlbÉRÈS René Marill (1966, 1972), Métamorphoses du roman, Albin Michel, Paris.

10. Cité d'après Martin-Achard, $2016: 125$. Revue wagnérienne, juin 1886. 
Archaimbault Sylvie (2000), « Un texte fondateur pour l'étude du dialogue : De la parole dialogale (L. Jakubinski), Histoire, Épistémologie, Langage, tome 22, fascicule 1, Horizons de la grammaire alexandrine, 99-115.

http://www.persee.fr/doc/hel_0750-8069_2000_num_22_1_2768

BALLET Gilbert (1886), Le Langage intérieur et les diverses formes d'aphasie, Alcan, Paris.

BANFIELD Ann ([1982] 1995), Unspeakable Sentences, trad. française Phrases sans paroles. Théorie du récit et du style indirect libre, Collection « Poétique », Édition du Seuil, Paris.

BAKHTine Mikhail ([1929] 1998), La Poétique de Dostoïevski, Collection « Points », Éditions du Seuil, Paris.

Bergounioux Gabriel (2001), « Endophasie et linguistiques [Décomptes, quotes et squelette] ", Langue française, $\mathrm{n}^{\circ} 132$, La parole intérieure, 106-107.

BERGOUNIOUX Gabriel (2001), «Esquisse d'une histoire négative de l'endophasie [avec une attention presque exclusive pour les productions en langue française consacrées à cette question] ", Langue française, $\mathrm{n}^{\circ} 132$, La parole intérieure, 3-25.

Bergounioux, Gabriel (2004), Le Moyen de parler, Verdier, Lagrasse.

BirCKetON, D. (1967), "Modes of Interior Monologue. A Formal Definition". Modern Language Quarterly, vol. 28, 229-239.

BowLING Lawrence Edward (1950), "What is Stream of Consciousness Technique?", $P M L A$, vol. 65. $\mathrm{n}^{\circ}$ 4, 333-345.

BrocA, Paul ([1861-1869] 2004), Écrits sur l'aphasie (1861-1869), L'Harmattan, Paris.

BÜHLER Karl ([1934] 2009), Sprachtheorie. Die Darstellungsfunktion der Sprache. [Das Organon-Modell], Verlag von Gustav Fischer, Iéna. Trad. française Théorie du langage, Agone.

Burunat Silvia (1980), El Monólogo interior como forma narrativa en la novela española 1940-1975, J. Porrúa Turanzas, Madrid.

CANnONE Belinda (2001), Narrations de la vie intérieure, Collection « Perspectives littéraires », PUF, Paris.

Carroy Jacqueline, OHAYON Annick et Plas Régine (2006), « Les origines de la psychologie française : Philosophie et médecine », Histoire de la psychologie en France XIX ${ }^{e}-X X^{e}$ siècles, La Découverte, Paris, 9-28. 
ChARDIN Philippe (dir.) (2004), Autour du monologue intérieur, Séguier/Atlantica, Paris/Biarritz.

Chrétien Jean-Louis (2009), Conscience et roman, 1. La conscience au grand jour, Collection « Paradoxe », Éditions de Minuit, Paris.

Chrétien Jean-Louis (2011), Conscience et roman : la conscience à mivoix, Collection « Paradoxe », Éditions de Minuit, Paris.

Coelho de Carvalho Alfredo Leme (1981), Foco narrativo e fluxo da consciência. questões de teoria literária, Pioneira, São Paulo.

CoHN Dorrit ([1978] 1981), Transparent Minds, trad. française La Transparence intérieure: Modes de représentation de la vie psychique dans le roman, Collection « Poétique », Éditions du Seuil, Paris.

CoHn Dorrit, The Distinction of Fiction ([1999] 2001), trad. française Le Propre de la fiction, Collection « Poétique », Éditions du Seuil, Paris.

Depretto Catherine (2012), De la « langue poétique » à la « parole dialogale » : retour sur Lev Jakubinskij (1892-1945) », Acta fabula, vol. 14, $\mathrm{n}^{\circ} 7$, « Ce qui a fait signe \& ce qui fait sens », octobre 2013, URL : http://www.fabula.org/acta/document8145.php, page consultée le 05 mai 2018.

DuJARDIN Édouard ([1887] 1991), The Bays are Sere and Interior Monologue, traduction et introduction par Anthony Suter, Libris, London.

DujARDIN Édouard (1931), Le Monologue intérieur. Son apparition, ses origines, sa place dans l'œuvre de James Joyce, Messein, Paris.

EgGER Victor ([1881] 1904), La Parole intérieure. Essai de psychologie descriptive, Alcan, Paris.

FODOR Jerry (1975), The Language of Thought, Harvard University Press, Cambridge.

FRANCEUR Louis (1976), « Le monologue intérieur narratif (sa syntaxe, sa sémantique et sa pragmatique) ", Études littéraires, vol. 9, $n^{\circ} 2,341-365$.

FRIEDRICH Janette (2001), « La discussion du langage intérieur par L.S. Vygostkij », Langue française, $n^{\circ} 132$, 57-71.

Friedman Melvin (1955), Stream of Consciousness. A Study in Literary Method, Yale University Press, New Haven.

Genette Gérard (1972), « Discours du récit », Figures III, Collection «Poétique », Éditions du Seuil, Paris.

Genette Gérard (1983), Nouveaux Discours du récit, Collection «Poétique », Éditions du Seuil, Paris. 
HAMBURGER Kate ([1977] 1986), Logique des genres littéraires, Collection « Poétique », Éditions du Seuil, Paris.

HumpHRey Robert (1968), Stream of consciousness in the Modern Novel, Collection "Perspectives in Criticism", University of California Press, Berkeley / Los Angeles.

IvanOVA Irene, SÉRIOT Patrick (2012), Lev Jakubinski, une linguistique de la parole (URSS, années 1920-1930), édition et traduction, Collection «Bilingues en sciences humaines », Lambert-Lucas, Limoges.

JAKOBSON Roman (1963), Études de linguistique générale 1 et 2, Éditions de Minuit, Paris.

JAKUBINSKI Lev P. (1923), La Parole dialogale, Léningrad.

JAMES William ([1890] 2002), "The Principles of Psychology" (trad. de l'anglais de la version abrégée de 1892) Précis de psychologie, Les empêcheurs de penser en rond, Paris.

JENNY Laurent (2002), " Méconnaissance du monologue intérieur ", La Fin de l'intériorité, Collection « Perspectives littéraires », PUF, Paris.

Joly André (1987), Essai de systématique énonciative, Presses universitaires de Lille, Lille.

LÄMMERT Eberhard (1955), Bauformen des Erzählens, Stuttgart.

LASSAGNE Laure (2016), Ce que se parler veut dire. La représentation du monologue dans les romans de Stendhal, Honoré Champion, Paris.

LETOUBLON François (2001), « Le discours et le dialogue intérieur chez Homère », M. Païsi-Apostolopoulou (éd.), Eranos, Center for Odyssean Studies, Ithaca.

MARTIN-ACHARD Frédéric (2010), « Les monologueurs de J. Serena : une colère 'objective' ?», Matteo Majorano (éd.), Écrire le fiel, B.A. Graphise, Bari, 45-58.

MARTiN-Achard Frédéric (2012), «Figures de l'intériorité dans le roman contemporain (F. Bon, L. Mauvignier, J. Serena) », Proses narratives en France au tournant du XXI ${ }^{e}$ siècle, 80-94.

MARTIN-ACHARD Frédéric (2015), «Imaginaires de la ponctuation et de l'endophasie », Littératures, Imaginaires de la ponctuation, $\mathrm{n}^{\circ} 72,51-66$.

MARTIN-ACHARD Frédéric (2016a), « Le langage de l'esprit à la croisée des chemins : Recherches littéraires, médicales et psychologiques 
sur le discours intérieur (1880-1900) ", L’Esprit Créateur, Vol. 56, $\mathrm{n}^{\circ} 4,118-131$.

MARTIN-ACHARD Frédéric (2016b), « Une intériorité sans psychologie ? Étude sur trois romans de la vie intérieure (Kerandal, Lenoir, Ndiaye) ", Revue critique de fiction française contemporaine (ISSN 2033-7019) : http://www.revue-critique-de-fixxion-francaise-contemporaine.org/rcffc/article/view/fx13.02

MARTIN-ACHARD Frédéric (2017), Voix intimes, voix sociales. Usages du monologue romanesque aujourd'hui, Collection «Études de littérature des XXe et XXI siècles », Classiques Garnier, Paris.

Mattia-Vivies Monique (2005), « Monologue intérieur et discours rapporté : parcours entre narratologie et linguistique », Bulletin de la société de stylistique anglaise, 9-24.

MATtia-Vivies Monique (2006), Le discours indirect en anglais contemporain : approche énonciative, Publications de l'Université de Provence.

MicheLET JACQUOT Valérie (2008), Le roman symboliste : Un art de l'« extrême conscience ». Édouard Dujardin, André Gide, Rémy de Gourmont, Marcel Schwob, Droz, Genève.

MoIx Gabrielle (1989), Valery Larbaud et l'évolution des formes littéraires, Peter Lang, Berne ; Francfort-s. Main ; Paris.

NetTeR Abraham ([1892] 2013), La Parole intérieure et l'âme, Hachette BNF, Paris.

PANACcio Claude (1999), Le Discours intérieur. De Platon à Guillaume d'Ockham, Éditions du Seuil, Paris.

PHILIPPe Gilles (1997), Le Discours en soi. La représentation du discours intérieur dans les romans de Sartre, Champion, Paris.

PhiLIPPE Gilles (2001a), « Le paradoxe énonciatif endophasique et ses premières solutions fictionnelles », Langue française, 132, 96-105.

PHILIPPE Gilles (2001b), «Quand et pourquoi peut-on parler d'un discours sans destinataire? ?, Énonciation et création littéraire. Actes du XIII colloque de l'ACLIF, Echinox, Cluj.

PHILIPpe Gilles (2009), « La langue littéraire, le phénomène et la pensée », Gilles Philippe et Julien Piat, (dir.), La Langue littéraire. Une histoire de la prose en France de Gustave Flaubert à Claude Simon, Fayard, Paris, 91-119.

Piaget Jean (1923), Langage et la pensée chez l'enfant, Delachaux et Niestlé, Paris. 
Puech Christian (2001), « Langage intérieur et ontologie linguistique à la fin du XXe siècle », Langue française, $\mathrm{n}^{\circ} 132,26-47$.

Proust Marcel (1919), À l'ombre des jeunes filles en fleurs, Gallimard, Paris.

Rabaté Dominique (1991), Vers une littérature de l'épuisement, Corti, Paris.

RABATEL Alain (2001), « La représentation de la parole intérieure [Monologue intérieur, discours direct et indirect libres, point de vue], Langue française, $\mathrm{n}^{\circ}$ 132, 72-95.

Rabatel Alain (2008), Homo narrans. Pour une analyse énonciative et interactionnelle du récit, Tome II : Dialogisme et polyphonie dans le récit, Lambert-Lucas, Limoges.

RAIMOND Michel (1985), La Crise du roman. Des lendemains du Naturalisme aux années vingt, José Corti, Paris, 257-298.

RosentHal Victor (2012), « La Voix de l'intérieur », Intellectica, 2012/2, $\mathrm{n}^{\circ} 58,53-89$.

Rosier Laurence (1999), Le Discours rapporté. Histoire, Théories, Pratiques, Collection «Champs linguistiques », Duculot, Gembloux.

SAInT-PAul Georges (1892), Essais sur le langage intérieur, Storck, Lyon.

SAINT-PAUl Georges (1904), Le langage intérieur et les paraphasies. La fonction endophasique, Alcan, Paris.

SALLENAVE Danièle (1972), « À propos du 'monologue intérieur'. Lecture d'une théorie », Littérature, n 5, 69-87.

SANTONE Laura (1998), “Victor Egger 'Annunciatore' di Édouard Dujardin e di James Joyce. Nuovi elementi sulla genesi del monologo interiore", Fin de siècle and Italy, Joyce Studies in Italy, 5, 245-278.

SANTONE Laura (1999), Voci dall'abisso: nuovi elementi sulla genesi del monologo interiore, Edipuglia, Bari.

SANTONE Laura (2009), Egger, Dujardin, Joyce: microscopia della voce nel monologo interiore, Roma, Bulzoni.

SCHOLES Robert (1966), The Nature of Narrative, New York, 178-188 et 283-299.

StANZEL Franz (1955), Die typischen Erzählsituationen in Roman dargestellt an Tom Jones, Moby-Dick, The Ambassadors, Ulysses, Wien.

StEINBERg Erwin Ray (1979), The Stream-of-Conciousness Technique in the Modern Novel, Kennikat Press, Port Washington, N.Y.; London. 
Struve Gleb (1954), "Monologue intérieur : The Origins of the Formula and the First Statements of its Possibilities", PMLA, vol. 69, $\mathrm{n}^{\circ}$ 5, 1101-1111.

VAN DER STAAY Elisabeth (1987), Le monologue intérieur dans l'auvre de Valery Larbaud, Champion/Slatkine, Paris/Genève.

VyGOTSKI Lev ([1934] 1997), Pensée et langage (1934) (traduction de Françoise Sève, avant-propos de Lucien Sève), suivi de «Commentaires sur les remarques critiques de Vygotski » de Jean Piaget, (Collection «Terrains», Éditions Sociales, Paris, 1985) ; Rééditions : La Dispute, Paris.

WelLeK René (1955), A History of Criticism 1750-1950, New Haven.

WeISMAN Frida (1978), Du monologue intérieur à la sous-conversation, Nizet, Paris.

WyZEWA Theodor (de) [1886-87] 1993, "Notes sur la littérature wagnérienne et les livres en 1885-1886 », Revue wagnérienne, 2, Slatkine Reprints, Genève, 9-21. 